\title{
Abordaje enfermero de la sexualidad en pacientes de salud mental de Asturias. Un estudio Delphi
}

\author{
Nursing Approach to Sexuality in Mental Health Patients in Asturias. A Delphi Study
}

\section{Mayte Palacio Roda, Natalia del Campo Robles}

Hospital San Agustín. Servicio de Salud Mental. Avilés (Asturias), España

Contacto: maytepalacioroda@gmail.com

Fecha de recepción: 24 de julio de 2019 / Fecha de aceptación: 2 de noviembre de 2019

\section{Resumen}

El presente estudio pretende aproximarse al abordaje de la sexualidad en pacientes de salud mental de Asturias, utilizando la técnica Delphi. Mediante el envío de tres cuestionarios sucesivos, se recabaron las opiniones de 29 enfermeros y enfermeras especialistas en salud mental. El primer cuestionario planteaba preguntas abiertas, en el segundo se presentaban una serie de afirmaciones para que los y las profesionales de enfermería mostraran su grado de acuerdo y en el tercero se pedía a los expertos que priorizaran las sentencias más relevantes.

Los resultados evidencian consenso en cuanto a que el abordaje que se realiza es escaso, debido a varios factores: falta de formación de los profesionales, de herramientas que guíen la exploración, así como de tiempo en la jornada laboral. Los enfermeros y enfermeras se sienten más cómodos abordando la sexualidad con pacientes de su mismo género. No se evidencia consenso respecto a la influencia de la edad o gravedad del diagnóstico en el abordaje de aspectos sexuales, pese a lo esperable comparándolo con otros estudios, en los que estos factores actúan como claros limitantes. Para mejorar la calidad de la atención sería necesaria la consideración de la sexualidad como un aspecto más del cuidado integral al paciente, el aumento de la formación específica en sexualidad y la realización de trabajo intrapersonal.

Palabras clave: sexualidad, salud mental, atención de enfermería, educación sexual, revisión por expertos, técnica Delphi.

\begin{abstract}
This study aims to approach the sexuality in mental health patients in Asturias, using the Delphi technique. By sending three successive questionnaires, the views of 29 mental health nurses were sought. The first questionnaire raised open questions, the second one made a series of statements for nursing professionals to show their degree of agreement and the third one asked the experts to prioritize the most relevant sentences.

The results show a consensus that the approach is limited, due to several factors: lack of training of professionals, lack of tools to guide exploration, and lack of time during working hours. Professionals feel more comfortable tackling sexuality with patients of their same gender. There is no consensus regarding the influence of the age or severity of the diagnosis on the approach to sexual aspects, despite what is expected compared to other studies, in which these factors act as clear limitations. In order to improve the quality of care, it would be necessary to consider sexuality as an aspect of comprehensive patient care, to increase specific training in sexuality and to carry out intra-personal work.
\end{abstract}

Keywords: sexuality, mental health, nursing care, sex education, peer review, Delphi technique.

\section{Introducción}

La sexualidad es un término relativamente reciente en el mundo sanitario, al menos no es hasta el año 2000 que la Organización Mundial de la Salud (OMS) estableció una definición oficial al respecto, en la que establece que se trata de "un aspecto fundamental de la condición humana, presente a lo largo de la vida que abarca el sexo, las orientaciones sexuales, las identidades de género, erotismo, así como la intimidad y la reproducción, entre otros"

La Confederación de Salud Mental Española en su Informe sobre el estado de los derechos humanos de 
las personas con trastorno mental realizado en 2017 , establece como una de sus recomendaciones promover el "empoderamiento de las personas con problemas de salud mental para que ejerzan libre, consciente y responsablemente su sexualidad y la no discriminación por su preferencia sexual"2.

Las personas con trastorno mental grave suelen presentar dificultades en la comunicación y falta de habilidades sociales, lo que afecta directamente a su capacidad para establecer relaciones y al manejo de su sexualidad. Estas dificultades se pueden ver aumentadas por el tratamiento psiquiátrico que en ocasiones requieren estos trastornos. Desde la Asociación Española de Sexualidad y Salud Mental (AESEXSAME), se han publicado múltiples investigaciones acerca de la disfunción sexual asociada a psicofármacos ${ }^{3}$.

Como se define en el Manual Diagnóstico y Estadístico de los Trastornos Mentales (DSM) ${ }^{4}$, las disfunciones sexuales son un "grupo de trastornos heterogéneos, que se caracterizan por la manifestación persistente de una alteración clínicamente significativa de la capacidad de la persona para responder sexualmente o para experimentar placer sexual". Las tasas de disfunciones sexuales son significativamente más altas en pacientes con problemas de salud mental que en la población general. La prevalencia estimada de trastornos sexuales en hombres tratados con psicofármacos es del $54 \%$, mientras que en las mujeres se reduce al 30\%. Los efectos que los antipsicóticos producen en las personas fueron analizados en 2015 en un estudio realizado por Marek Just ${ }^{5}$, que establece como principales problemas para el hombre el conseguir y mantener la erección, los trastornos de la eyaculación, la disminución de la intensidad del orgasmo y la aparición de priapismo. En cuanto a las mujeres tratadas con psicofármacos, se observó por su parte la dificultad para alcanzar el orgasmo y el dolor durante el mismo, la hiperprolactinemia y la galactorrea. En ambos sexos se produce también una importante disminución del apetito sexual.

La aparición de estos efectos secundarios indeseables afecta a aspectos fundamentales de la vida, entre ellos la reproducción y las relaciones, produciendo un menor bienestar subjetivo, disminución de la calidad de vida, poca satisfacción física y emocional. Además, afecta al cumplimiento terapéutico, ocasionando en un significativo número de pacientes el abandono de la medicación y la reaparición de los síntomas psicóti$\cos ^{6}$.
En cuanto a la implicación de los y las profesionales en el estudio de la sexualidad de los pacientes de salud mental, las primeras publicaciones son realizadas a partir de comienzos del siglo XXI. Hasta ese momento destaca la falta de literatura publicada sobre el tema, lo que manifiesta el poco interés que se presentó hasta ese momento por la sexualidad de estos pacientes. Sin embargo, en estos últimos 15 años, se ha desarrollado una mayor preocupación por explorar este tema. Ha crecido exponencialmente el número de publicaciones al respecto en forma de revisiones y estudios sobre sexualidad, enfermería y salud mental ${ }^{7}$.

Antes de que los y las profesionales de enfermería puedan ayudar a otros con sus necesidades sexuales y afectivas, es posible que necesiten examinar sus propios valores, miedos y creencias. Las propias actitudes de las enfermeras y los enfermeros con respecto a la sexualidad pueden actuar como una barrera para la exploración de problemas sexuales con los pacientes. Además, debe haber una actitud consciente y proactiva por su parte para proporcionar educación sexual y fomentar la exploración de los sentimientos y recursos de los pacientes, ya que las necesidades de sexualidad no suelen ser demandadas de forma espontánea por los usuarios.

Los y las profesionales de enfermería pueden actuar evitando abordar el tema de la sexualidad con los pacientes porque consideren que hay otras funciones más importantes o que la sexualidad no es una prioridad en el tratamiento de los pacientes. Además, puede que no lo consideren como parte de sus responsabilidades o que no se encuentren capacitadas para hacer frente a estos problemas de índole sexual, como recoge Quinn en su estudio publicado en $2009^{7}$. Es por esto que, muchas veces, no se aborda espontáneamente la sexualidad en los pacientes con problemas de salud mental, sino que espera que sean los pacientes los que les proporcionen alguna indicación de que están experimentando un problema en ese ámbito para tratar el tema o, en otro caso, ante una demanda, actúan derivándola a otro profesional ${ }^{8,9}$.

Los y las pacientes, por su parte, manifiestan que no reciben apoyo suficiente por parte de las enfermeras para manejar sus dificultades a la hora de establecer relaciones sexuales seguras y satisfactorias ${ }^{10,11}$.

Para mejorar este aspecto de la atención, es necesaria una buena educación en sexualidad para los profesionales acompañada de un impulso por parte de la 
organización para fomentar el abordaje. Destaca también la importancia del uso de herramientas específicas en el abordaje de la sexualidad, las cuales han sido probadas en diferentes estudios, como los realizados por Quinn ${ }^{12-14}$ o el realizado por Salked en $2015^{15}$, obteniendo resultados satisfactorios y reconociendo por parte del equipo de enfermería una mayor confianza en sí mismos y una mayor implicación.

Tras revisar la literatura publicada respecto al abordaje de enfermería en la sexualidad de las personas diagnosticadas de problemas de salud mental, observamos que las actitudes y la formación y capacitación de los y las profesionales parecen jugar un papel decisivo. Apenas hemos encontrado en España publicaciones que incluyan los tres aspectos claves de nuestro estudio: sexualidad, enfermería y salud mental. Por todo ello nos planteamos conocer las actitudes y las opiniones de los y las profesionales de enfermería sobre el abordaje de la esfera sexual y por qué este aspecto no suele formar parte de la práctica diaria, lo que nos permitirá desarrollar estrategias que faciliten las intervenciones de enfermería a este respecto.

\section{Material y métodos}

Se ha llevado a cabo un estudio de tipo descriptivo utilizando la técnica Delphi, la cual se consideró más adecuada para la aproximación al consenso de un grupo de expertos basado en el análisis y la reflexión sobre el abordaje de la sexualidad en los pacientes de salud mental. El ámbito del estudio fue el Servicio de salud mental del Principado de Asturias que abarca los siguientes dispositivos distribuidos en las siete Áreas de Salud de la Comunidad Autónoma:

- 5 Unidades de Hospitalización Psiquiátrica de adultos (UHP).

- 1 Unidad de Hospitalización Psiquiátrica Infanto/Juvenil (UHP-IJ).

- 16 Centros de Salud Mental de Adultos (CSM).

- 4 Centros de Salud Mental Infanto/Juveniles (CSM-IJ).

- 4 Unidades de Tratamiento de Toxicomanías (UTT).

- 2 Hospitales de Día de Trastorno de la Conducta Alimentaria (HD-TCA).

- 8 Hospitales de Día de trastornos mentales severos (HD).

- 2 Equipos de Tratamiento Asertivo Comunitario (ETAC).

- 8 Comunidades Terapéuticas (CT).
Se seleccionaron un total de 59 expertos para formar parte del grupo de consenso, de los cuales finalmente aceptaron participar 34. Uno de los criterios de inclusión que se consideró fue que tuviesen la titulación de enfermeros/as especialistas en salud mental y que desarrollasen su profesión dentro del Servicio Asturiano de Salud en el momento del estudio, con independencia del área o dispositivo de trabajo. Otro de los criterios utilizados para la selección de los expertos fue que hubieran participado de forma activa en grupos de trabajo, comisiones y/o actividades docentes. Con todos ellos se contactó previamente mediante correo electrónico para solicitar su colaboración en el estudio, informando brevemente sobre los objetivos y las características de este y sobre el carácter anónimo de sus respuestas. Una vez obtenida la confirmación de los expertos, se comenzó la recogida de datos, la cual se desarrolló entre diciembre de 2018 y febrero de 2019.

Se llevó a cabo en tres fases mediante el envío de sucesivos cuestionarios vía correo electrónico, en los cuales se indicaron las correspondientes instrucciones de cumplimentación, así como el plazo de respuesta. En el correo electrónico se adjuntó un enlace online donde se cumplimentaría el cuestionario, garantizando el anonimato de las respuestas. Tres días antes del vencimiento del plazo se les envió, en cada una de las fases, un correo electrónico a todos los participantes a modo de recordatorio.

La primera fase consistió en una serie de preguntas abiertas de opinión libre que invitaban a la reflexión. A los encuestados se les pidió que respondieran de forma detallada y precisa, evitando en lo posible monosílabos y frases estereotipadas. Las cuestiones que conformaron la primera fase se muestran a continuación:

1. En tu opinión, ¿cómo crees que se realiza por parte de los profesionales de enfermería el abordaje de la sexualidad en los pacientes de salud mental?

2. ¿Qué características de los profesionales de enfermería crees que favorecen el abordaje de la sexualidad?

3. ¿Cuáles crees que son las principales dificultades relativas a las actitudes, valores y creencias generales de los profesionales que se dan en la práctica diaria y que pueden influir en el modo de concebir y abordar la sexualidad por parte de los profesionales de enfermería?

4. ¿Cuáles crees que son las principales dificultades relativas a las características del paciente (edad, 
género, diagnóstico) que se dan en la práctica diaria y que pueden influir en el modo de abordar la sexualidad por parte de los profesionales de enfermería?

5. ¿Cuáles crees que son las principales dificultades relativas al contexto (tipo de dispositivo, disponibilidad de tiempo, herramientas informáticas) que se dan en la práctica diaria y que pueden influir en el modo de abordar la sexualidad por parte de los profesionales de enfermería?

6. ¿Qué factores consideras que influyen en que los pacientes expresen o no sus necesidades o demandas en relación con la sexualidad a los profesionales de enfermería?

7. ¿Qué intervenciones consideras que se deberían realizar por parte de los profesionales de enfermería para prevenir las conductas sexuales de riesgo en pacientes de salud mental?

8. ¿Qué crees que se podría hacer para mejorar la atención a estos pacientes en materia de sexualidad?

Junto a este primer cuestionario se adjuntó un enlace para la cumplimentación de una pequeña plantilla de recogida de datos sociodemográficos. Cada una de las investigadoras llevó a cabo un análisis detallado de las respuestas del primer cuestionario, extrayendo de ellas una serie de sentencias que reflejaran las respuestas del grupo. A continuación, se pusieron en común ambos listados de sentencias resultando un total de 48 sentencias, divididas en ocho epígrafes, que se reflejaron en el segundo cuestionario. En el segundo cuestionario se solicitó a los expertos que expresaran su grado de acuerdo con las sentencias mediante una escala tipo Likert con cinco opciones a las que se asignó un valor numérico. Se insistió en que evitaran otorgar, de forma automática, la misma puntuación a todas las respuestas. Las sentencias se dispusieron de forma ordenada y categorizada en relación con las preguntas del cuestionario uno.

Para analizar las respuestas a este segundo cuestionario se calculó la puntuación media en la escala Likert, sumando las puntuaciones que recibió cada sentencia y dividiéndolo entre el número total de respuestas, obteniendo así un valor que oscilaría entre 1 y 5 , de acuerdo con las cinco opciones de la escala Likert. Se consideró que los valores comprendidos entre 1-2,5 expresaban desacuerdo, los comprendidos entre 2,5-3,5 mostraban indiferencia/división de opiniones y los comprendidos entre 3,5-5 reflejaban acuerdo. En el tercer cuestionario se presentaron las sentencias del segundo cuestionario junto con la puntuación media obtenida. Con esta información se pidió a los expertos que seleccionaran, para cada uno de los ocho epígrafes iniciales, las tres sentencias que consideraran más relevantes, clasificándolas según su prioridad (primera, segunda y tercera).

Del análisis de este cuestionario se obtuvo la prioridad media de cada una de las sentencias, que se calculó otorgando tres puntos a las sentencias con primera prioridad, dos puntos a las sentencias de segunda prioridad y un punto a las sentencias de tercera prioridad, y dividiendo el resultado entre el total de respuestas. De esta forma se obtuvieron valores comprendidos entre 0 y 3 , correspondiendo los valores más altos a las sentencias más priorizadas según la opinión de los expertos. Del análisis de estos datos se extrajeron conclusiones sobre la percepción del abordaje de la sexualidad en pacientes de salud mental por parte de los profesionales de enfermería especialistas en salud mental de Asturias.

\section{Resultados}

De las 34 personas que accedieron a participar en el estudio, 29 contestaron al primer cuestionario $(85,3 \%), 23$ al segundo $(67,6 \%)$ y 19 al tercero $(55,9 \%)$. Respecto a los datos demográficos reflejados por el grupo de expertos, destacamos que hubo una notable diferencia en cuanto al sexo ya que el $89,6 \%$ eran de sexo femenino y el $10,3 \%$ de sexo masculino. Se obtuvo una media de edad de 44,5 años, en un rango que se presentaba entre 30 y 59 años. La distribución de los participantes por área de trabajo en el momento actual reflejó una mayoría de participantes por parte de las áreas centrales de Avilés, Oviedo y Gijón, que en conjunto representaron el $86,2 \%$ del total, en detrimento de las áreas periféricas de Jarrio, Arriondas y Mieres que representaron el 13,8\% del total. No se obtuvieron representantes de las áreas de Cangas del Narcea y Langreo. Respecto al dispositivo de trabajo actual, la mayor parte del grupo de expertos se identificaron como trabajadores del centro de salud mental $(27,5 \%)$. En cuanto a los años de experiencia totales de los participantes como profesionales de enfermería, se obtuvo una media de 22,6 años, y concretamente como enfermeros especialistas, una media de 15,9 años.

Del análisis de las respuestas al primer cuestionario se extrajeron 48 sentencias, 6 para cada una de las 8 preguntas. Para cada sentencia se calculó una puntuación media en base al segundo cuestionario (Anexo 1) 
y un índice de priorización resultante del tercer cuestionario (Anexo 2). Respecto a la primera cuestión referida al abordaje de la sexualidad por parte de los y las profesionales de enfermería, destacaron las sentencias que sugieren que el abordaje es escaso y solo se realiza ante una demanda específica del paciente, con unas puntuaciones medias elevadas $(4,6$ y 4,2$)$. Coherentemente con estas afirmaciones, la sentencia que manifestó un buen abordaje de la sexualidad obtuvo la puntuación media más baja $(1,4)$, lo que refleja un claro desacuerdo grupal con la misma. En el primer cuestionario, de respuesta libre, se obtuvieron varias opiniones que coincidían en que uno de los factores que determina el escaso abordaje de la sexualidad por parte de los y las profesionales de enfermería es que estos no se ven capacitados o bien creen que su formación es escasa; esta afirmación fue recogida en el cuestionario número dos y obtuvo una puntuación media elevada $(4,6)$, lo que demuestra un alto grado de acuerdo entre los expertos. Otro de los factores determinantes que se expusieron en el primer cuestionario fue la falta de apoyo que los y las profesionales reciben por parte del personal médico, aunque esta sentencia no suscitó tanto acuerdo entre los expertos y las expertas. Sobre el conjunto de respuestas relativas a las características de los y las profesionales que favorecen el abordaje de la sexualidad, destaca el amplio acuerdo en torno a las cualidades de accesibilidad, disponibilidad, asertividad y flexibilidad, así como a la relación terapéutica continuada, con puntuaciones medias superiores a 4 . Los resultados del estudio reflejan así mismo que el abordaje de la sexualidad está influido por las actitudes y creencias de los y las profesionales hacia su propia sexualidad, existiendo aún tabús y prejuicios con respecto al sexo. Los expertos se mostraron ampliamente de acuerdo con la sentencia que afirmó que se minusvalora la importancia de una vida sexual satisfactoria en los pacientes de salud mental, siendo la sentencia más priorizada en este grupo.

Relativo a las características del paciente, existió diversidad de opiniones en cuanto a la relación entre el abordaje de la sexualidad y la gravedad del diagnóstico del paciente o su edad. Sin embargo, sí parece existir consenso en cuanto al género, ya que los expertos refirieron sentirse más cómodos abordando la sexualidad con pacientes de su mismo género, recibiendo esta sentencia una puntuación media de 4,3. Cabe destacar que los expertos y expertas priorizan como dificultades relativas al contexto la falta de tiempo en la jornada laboral y la falta de herramientas informáticas específicas que guíen la exploración de la sexualidad. También resultó llamativa la división de opiniones en cuanto a si los pacientes suelen realizar o no demandas relacionadas con su sexualidad, lo que se ve reflejado en una puntuación media de 2,7 para esta sentencia. En cambio, existe consenso en cuanto a que la falta de información sobre sexualidad que reciben los pacientes por parte de los profesionales resulta determinante para que estos no realicen demandas al respecto, sentencia con una puntuación media de 4,3.

El grupo de sentencias relativas a las conductas sexuales de riesgo es el que ofrece un mayor consenso general. Por un lado, los expertos y expertas coinciden en la necesidad de realizar intervenciones de educación sexual por parte de los profesionales de enfermería como medida de prevención. Por otro lado, manifiestan la opinión de que actualmente estas intervenciones son muy escasas en la práctica clínica. Como ideas de mejoras en la atención a los pacientes de salud mental en materia de sexualidad, los expertos y expertas coincidieron en que es prioritario dar más formación en este campo a los profesionales de enfermería, así como realizar un trabajo intrapersonal analizando los propios valores y creencias, con el objetivo de que la sexualidad forme parte del cuidado integral de los pacientes de forma habitual.

\section{Discusión}

En términos generales, los resultados del estudio describen un escaso abordaje de la sexualidad por parte de los y las profesionales de enfermería, lo que coincide con otros estudios realizados en este mismo campo. El grupo de expertos y expertas manifestó que uno de los motivos por el que este abordaje no se lleva a cabo de forma habitual es la falta de formación específica de los enfermeros y las enfermeras sobre sexualidad. Este dato resulta coherente con la literatura revisada, ya que diversos estudios destacan la formación como factor determinante en la disposición de los y las profesionales para abordar la sexualidad. Resulta llamativo que en el año 2019 la falta de formación siga siendo un factor limitante, cuando ya en el año 2000 la OMS estableció como una de sus metas la realización de un programa de capacitación para dar educación y apoyo a los y las profesionales sanitarios, necesario para promover eficazmente la salud sexual ${ }^{1}$.

Parece existir un amplio consenso en cuanto a que la sexualidad solo se aborda cuando un paciente realiza alguna demanda específica al respecto. Este dato contrasta con las recomendaciones de otros estudios 
publicados en los que se hace hincapié en la necesidad de realizar una exploración proactiva de los aspectos relacionados con la sexualidad ${ }^{20}$. Aunque en la primera fase del estudio algunos expertos manifestaron el escaso apoyo del personal médico como uno de los motivos que limitaban el abordaje de enfermería en materia de sexualidad, no se produjo un consenso por parte del grupo en la segunda fase. No se encontraron referencias claras al respecto en la literatura revisada, por lo que sería interesante la profundización en este tema en futuras investigaciones, para valorar mejor las relaciones de equipo entre profesionales sanitarios.

Como se observa en los estudios de Quinn y Happell $^{13}$, es habitual que existan dudas en referencia a si el abordaje de la sexualidad forma parte de las competencias de enfermería, sin embargo, el presente estudio refleja un claro consenso en este aspecto. Los expertos y las expertas reconocen el abordaje de la sexualidad como una de las competencias enfermeras, no siendo necesaria una orden médica previa para la intervención. Es posible que esta discordancia sea debida a que la mayoría de estudios revisados fueron llevados a cabo en otros países en los que las competencias que definen la profesión enfermera pueden diferir de las asumidas en España.

Una de las categorías a las que hace referencia este estudio trata sobre los valores y creencias de los y las profesionales de enfermería sobre su propia sexualidad y cómo estos pueden influir en sus intervenciones. A este respecto, los expertos y las expertas coinciden en que aún existen algunos tabúes y prejuicios en torno a la sexualidad por parte de los profesionales, aunque el consenso es menor respecto a la influencia de estas creencias en la forma de abordar la sexualidad. La tendencia de la literatura revisada es a considerar estas percepciones subjetivas cada vez menos presentes en los ámbitos científicos, sustituyéndose por datos científicos y actitud profesional en el tratamiento de la sexualidad, orientaciones y prácticas sexuales ${ }^{12}$.

Respecto a las características de los pacientes que influyen en el abordaje de la sexualidad, son muchos los artículos que afirman que los y las profesionales de enfermería se encuentran más cómodos cuando el paciente es una persona perteneciente a su mismo sexo $^{7}$. Este dato coincide con lo recogido en este estudio, siendo la concordancia de género entre el profesional y el paciente la característica que más consenso suscitó por parte de los expertos.
Existen otros factores que influyen en el abordaje de la sexualidad englobados en el contexto en el que se desarrolla la práctica asistencial. Las tres sentencias con más consenso de este grupo hacen referencia a la falta de tiempo en la jornada laboral para abordar la sexualidad, la falta de herramientas informáticas específicas que guíen la exploración y el tipo de dispositivo en el que se encuentre el paciente. Estos resultados se muestran de acuerdo a lo esperado, ya que estudios de opinión realizados en otros países, afirman que la carga asistencial, especialmente en unidades de agudos, hace que se prioricen cuestiones más acuciantes para la salud y se minimice o incluso se anule por completo la atención a las necesidades sexuales de los pacientes ${ }^{13}$.

Respecto a las herramientas que pueden servir como guía en la exploración, no se hace referencia específica en la literatura a las de tipo informático. Sí son en cambio numerosos los estudios que valoran la eficacia de la implementación de guías o protocolos para favorecer el abordaje de la sexualidad por parte de los y las profesionales de enfermería, obteniendo resultados muy satisfactorios, reconociendo por parte del equipo de enfermería una mayor confianza en sí mismos/as y una mayor implicación. Uno de los modelos propuestos es el PLISSIT, acrónimo que procede de las iniciales en inglés de las fases de dicho modelo: "Permiso", "Información Limitada", "Sugerencias Específicas" y "Terapia Intensiva". De la misma manera, otros de los modelos más conocidos, el denominado BETTER, recibe este nombre por sus fases, que son las siguientes: "Sacar", "Explicar", "Decir", “Tiempo", "Educar" y "Recordar" $^{, 6,12,18}$. Sería interesante ampliar las investigaciones en esta dirección en España, implantando la utilización de alguno de estos modelos y valorando posteriormente su eficacia.

En la categoría de preguntas que hacen referencia a los factores que influyen en que los pacientes expresen o no sus necesidades sexuales, el consenso fue casi pleno respecto a la importancia de una relación terapéutica de confianza entre paciente-enfermero para facilitar la expresión de sentimientos y aspectos íntimos. En la mayoría de las herramientas específicas que sirven como guía para la exploración de la sexualidad hay una fase consistente en explicar la sexualidad, dar información u ofrecer consejo sanitario, así como dar un tiempo para la reflexión y posponer el abordaje al momento que el paciente considere adecuado ${ }^{19}$.

La última categoría de sentencias se compone de sugerencias y posibles estrategias de mejora para 
alcanzar una atención de enfermería de calidad. En este aspecto, la sentencia que roza el consenso total es la necesidad de aumentar o mejorar la formación en materia de sexualidad que los profesionales reciben. Siguiendo a esta en orden de puntuación, se hace alusión a que se debería tratar la sexualidad como un aspecto más de la valoración enfermera y del cuidado integral a los pacientes ${ }^{20,13}$.

La elección de la técnica Delphi resulta adecuada para esta investigación, ya que, como se observa en la literatura, la sexualidad tiene un componente subjetivo y moral. "En las disciplinas no exactas, en situaciones de incertidumbre o cuando se carece de información objetiva es apropiado utilizar como recurso el juicio subjetivo de expertos" ${ }^{\text {"14 }}$. En cuanto al número de expertos y expertas, varios artículos consultados establecen como adecuado un grupo entre 15 y 25 miembros, otros lo reducen incluso a 10-18 ${ }^{15,16}$. En este estudio los participantes oscilaron entre 29 en el primer cuestionario y 19 en el tercero, por lo que se considera una muestra aceptable y representativa.

Este estudio presenta algunas limitaciones, como la subjetividad en la realización de las preguntas del primer cuestionario, el cual se realizó basándose en la literatura revisada y puede estar influido por las opiniones de las investigadoras, así como la realización de los ítems del segundo cuestionario. Otra limitación es que no se realizó un cálculo del tamaño muestral. En cuanto a la selección de los expertos, que se concentraron en las tres áreas principales del principado de Asturias, es esperable que se trate de un grupo homogéneo por lo que la validez interna del estudio sería adecuada; aun así, los resultados puede que no sean aplicables al resto del territorio español.

\section{Conclusiones}

Del análisis de las opiniones recogidas en este estudio por parte de los y las profesionales de enfermería se concluye que actualmente el abordaje de la sexualidad en los pacientes de salud mental de Asturias es escaso y se encuentra altamente influido por ciertos factores como la diferencia de género con el paciente o los propios valores y creencias de los enfermeros y enfermeras. Estos y estas son conscientes de la importancia de la sexualidad en los pacientes de salud mental como parte de la atención integral, sin embargo, aún no se ha implementado este cuidado como uno más de los que los y las profesionales de enfermería brindan a los pacientes en la práctica diaria. Es necesario un esfuer- zo consciente por parte de los y las profesionales actuando de manera proactiva para asegurar que los pacientes tengan acceso a información sobre sexualidad, así como para realizar evaluaciones periódicas que guíen a los pacientes hacia una salud sexual óptima y que otorguen a estos la seguridad y el apoyo para mantener relaciones íntimas sanas y satisfactorias. También es necesario un impulso por parte de la organización que apoye a los y las profesionales de enfermería en el abordaje de la sexualidad y que les brinde las herramientas y la formación adecuadas para que los y las profesionales se sientan más cómodos y capacitados para el desempeño de este tipo de intervenciones.

\section{Bibliografía}

1. Organización Panamericana de la Salud (OPS) Organización Mundial de la Salud (OMS). Conferencia: promoción de la salud sexual. Recomendaciones para la acción. Antigua GuatemalaGuatemala, 19-22 Mayo, 2000. 64p

2. Informe sobre el estado de los derechos humanos de las personas con trastorno mental en España. Confederación Salud Mental España. 2017 [acceso 19 de septiembre de 2018]. Disponible en: https://www.consaludmental.org/publicaciones/InformeDerechos-Humanos-Salud-Mental-2017.pdf.

3. Grupo Español de Trabajo para el Estudio de las Disfunciones Sexuales Secundarias a Psicofármacos. Propiedades psicométricas del Cuestionario de Disfunción Sexual Secundaria a Psicofármacos. Actas Esp Psiquiátr. 2000; 28(3): 141-150.

4. Asociación Americana de Psiquiatría (APA). Manual Diagnóstico y Estadístico de los Trastornos Mentales DSM-5. $5^{\text {a }}$ ed. Editorial Médica Panamericana. Madrid. 2014 [acceso 23 de agosto de 2018]. Disponible en: https://dsm.psychiatryonline.org/

5. Just MJ. The influence of atypical antipsychotic drugs on sexual function. Neuropsychiatr Dis Treat. 2015; 11:1655-1661.

6. Hurtado F, Domínguez O. Vademecum sexual: Fármacos y disfunción sexual. Psicosom Psiquiatr. 2017; 1(1): 27-59.

7. Quinn C, Browne G. Sexuality of people living with a mental illness: A collaborative challenge for mental health nurses. Int J Ment Health Nurs. 2009; 18: 195-203.

8. Quinn C, Happell B, Browne G. Talking or avoiding? Mental health nurses' views about discussing sexual health with consumers. Int J Ment Health Nurs. 2011; 20: 21-28.

9. Mccann E. Investigating mental health service user views regarding sexual and relationship issues. J Psychiatr Ment Health Nurs. 2010; 17: 251-259.

10. Quinn C, Dip G, Happell B. Sex on show. Issues of privacy and dignity in a Forensic mental health hospital: Nurse and patient views. J Clin Nurs. 2015; 24: 2268-2276.

11. Quinn C, Happell B. Talking About Sexuality With Consumers of Mental Health Services. Perspect Psychiatr Care. 2013; 49: 1320

12. Quinn C, Happell B, Welch A. The 5-As Framework for Including Sexual Concerns in Mental Health Nursing Practice. Issues Ment Health Nurs. 2013; 34: 17-24.

13. Quinn C, Happell B, Welch A. Talking about sex as part of our role: Making and sustaining practice change. Int J Ment Health Nurs. 2013; 22: 231-240.

14. Quinn C, Happell B. Getting BETTER: Breaking the ice and warming to the inclusion of sexuality in mental health nursing care. Int J Ment Health Nurs. 2012; 21:154-162.

15. Salkeld J. Assessing sexual health in mental health service users. Nursing Standard. 2015;30(5): 53-58. 
16. Varela-Ruiz M, Díaz-Bravo L, García-Durán R. Descripción y usos del método Delphi en investigaciones del área de salud. Inv Ed Med. 2012; 1(2):90-95.

17. Yañez $R$ y Cuadra R. La técnica Delphi y la investigación en los servicios de salud. Cienc Enferm. 2008; 14(1): 9-15.

18. García-Valdés $M$, Suarez-Marín M. Empleo del método Delphi en investigaciones sobre salud publicadas en revistas científicas cubanas. Rev Cuba Inf Cienc Salud. 2013;24(2): 1-12.

19. Cort EM, Attemborough J, Watson JP. An initial exploration of community mental health nurses attitudes to an experience of sexuality-related issues in the work with people experiencing mental health problems. J Psychiatr Ment Health Nurs. 2001; 8: 489-499.

20. Mick JM. Sexuality assessment: 10 strategies for improvement. Clin J Oncol Nurs. 2007; 11(5): 671-675.

21. Higgins A, Barker P, Begley CM. 'Veiling sexualities': a grounded theory of mental health nurses responses to issues of sexuality. $J$ Adv Nurs. 2008; 62(3): 307-317.

22. Navarro JI, Rodríguez C, Garrido P, De la Rosa E, Moreno A et al. Valoración del Patrón de la Sexualidad: atención integral de la salud de las personas. Enf Glob. 2013; 12(31): 1-13. 


\section{Anexos}

Anexo 1. Distribución de las respuestas a la escala Likert del cuestionario dos.

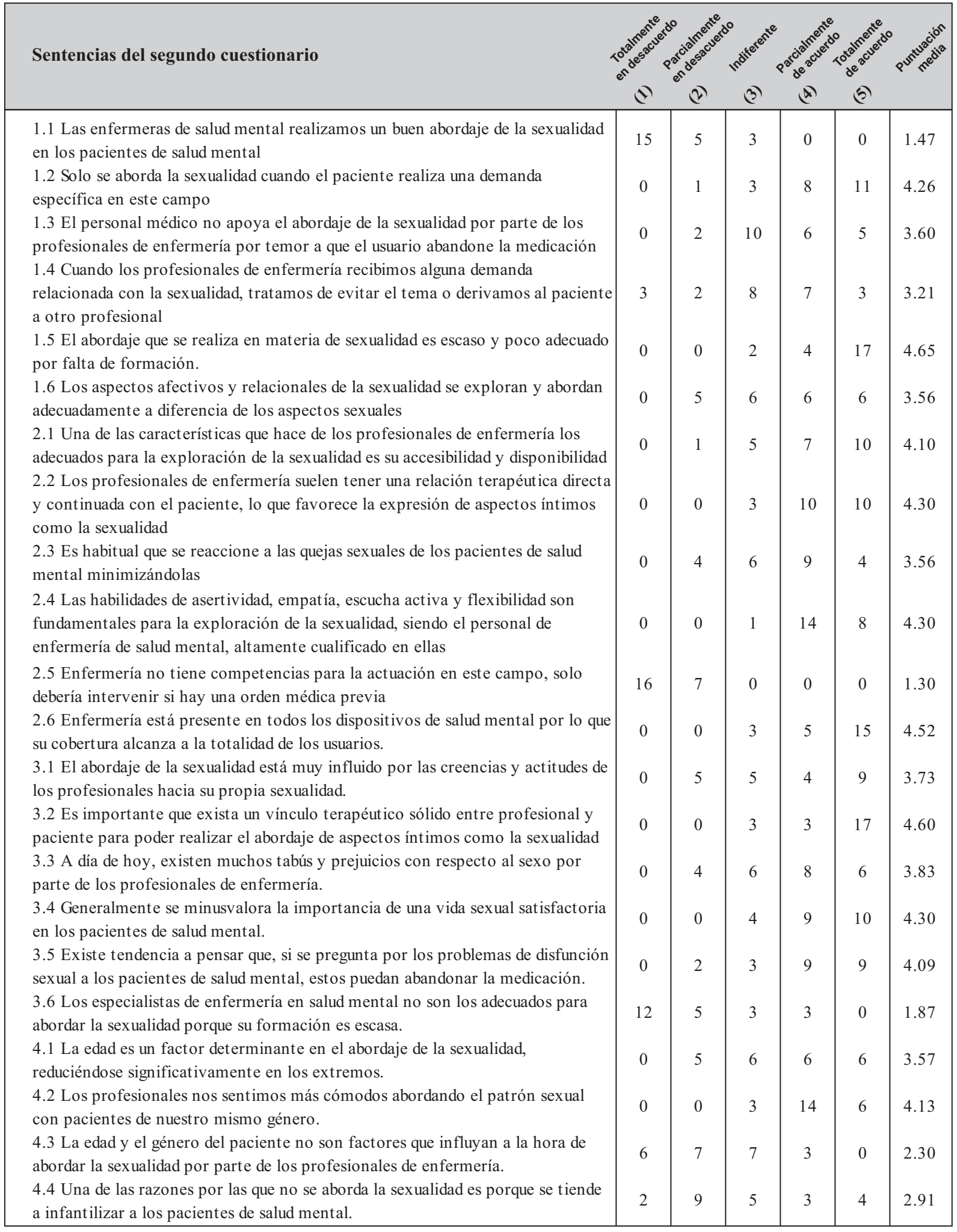


4.5 Los pacientes con diagnóstico de psicosis son menos preguntados por la sexualidad que pacientes con diagnósticos mentales menos graves.

4.6 Las características del paciente no influyen en el abordaje de la sexualidad.

5.1 No se disponen de herramientas informáticas elaboradas que guíen la exploración de la sexualidad por parte de los profesionales de enfermería. 5.2 La falta de tiempo en la jornada laboral hace que los profesionales de enfermería prioricemos otros aspectos de la enfermedad antes de la sexualidad. 5.3 El tipo de dispositivo en el que desarrollamos nuestro trabajo es el factor contextual que más influye a la hora de abordar la sexualidad.

5.4 Los dispositivos de rehabilitación son los más adecuados para abordar la sexualidad de los pacientes.

5.5 La falta de espacios físicos que garanticen la intimidad y la confidencialidad del paciente hace que no se aborde la sexualidad.

$5.6 \mathrm{El}$ contexto en general no influye en que los profesionales enfermeros aborden o no la sexualidad de los pacientes.

6.1 Los pacientes de salud mental no suelen hablar de sexualidad porque no es un aspecto relevante para ellos.

6.2 Los pacientes de salud mental si demandan atención cuando tienen algún problema de índole sexual.

6.3 La falta de confianza por parte del paciente con su enfermero, hace que no demande atención relacionada con la sexualidad.

6.4 La falta de información de los profesionales a los pacientes sobre sexualidad hace que los pacientes no realicen demandas al respecto.

6.5 Los pacientes de salud mental asumen los problemas con la sexualidad como parte de su enfermedad y no creen que los profesionales enfermeros puedan ayudarlos.

6.6 Una buena relación terapéutica facilita al paciente la expresión de aspectos relacionados con la sexualidad.

7.1 Los pacientes de salud mental establecen relaciones sexuales de riesgo con más frecuencia que la población general.

7.2 Los profesionales de enfermería apenas realizan intervenciones dirigidas a prevenir las conductas sexuales de riesgo en los pacientes de salud mental.

7.3 La educación sexual es la base para evitar conductas sexuales de riesgo en los pacientes de salud mental.

7.4 Es necesario realizar talleres grupales específicos para abordar las conductas sexuales de riesgo.

7.5 El reparto de preservativos en los dispositivos y la información sobre métodos anticonceptivos son medidas eficaces para disminuir las conductas sexuales de riesgo.

7.6 Se podrían reducir los embarazos no deseados en pacientes de salud mental con estrategias de prevención de conductas sexuales de riesgo.

8.1 Para mejorar la atención de los pacientes en materia de sexualidad se debe dar información sobre el tema a todos los pacientes.

8.2 La sexualidad de los pacientes de salud mental no es un aspecto que precise mejoras ya que no es un objetivo en la atención.

8.3 Para mejorar la atención de los pacientes en materia de sexualidad se debe tratar esta como un aspecto más de la valoración integral.

8.4 Se debe dar más formación al personal de enfermería para concienciar de la importancia de la sexualidad en los pacientes.

8.5 Es necesario realizar un trabajo intrapersonal, analizando nuestros propios valores y creencias, para mejorar la atención en sexualidad.

8.6 Hay pocas intervenciones que los profesionales de enfermería podamos

desarrollar para mejorar la atención en sexualidad.

\begin{tabular}{|c|c|c|c|c|c|}
\hline 0 & 2 & 12 & 5 & 4 & 3.48 \\
\hline 18 & 3 & 1 & 1 & 0 & 1.35 \\
\hline 0 & 2 & 5 & 5 & 11 & 4.09 \\
\hline 0 & 0 & 2 & 6 & 15 & 4.56 \\
\hline 0 & 0 & 0 & 10 & 13 & 4.57 \\
\hline 0 & 7 & 4 & 7 & 5 & 3.43 \\
\hline 2 & 2 & 5 & 9 & 5 & 3.57 \\
\hline 15 & 5 & 3 & 0 & 0 & 1.73 \\
\hline 9 & 13 & 1 & 0 & 0 & 1.65 \\
\hline 2 & 12 & 3 & 3 & 3 & 2.7 \\
\hline 0 & 2 & 4 & 4 & 13 & 4.22 \\
\hline 0 & 0 & 3 & 10 & 10 & 4.30 \\
\hline 7 & 3 & 1 & 5 & 7 & 3.09 \\
\hline 0 & 0 & 0 & 7 & 16 & 4.70 \\
\hline 0 & 0 & 3 & 10 & 10 & 4.30 \\
\hline 0 & 6 & 6 & 9 & 2 & 3.83 \\
\hline 0 & 0 & 0 & 4 & 19 & 4.83 \\
\hline 0 & 3 & 0 & 4 & 16 & 4.43 \\
\hline 2 & 1 & 5 & 5 & 10 & 3.87 \\
\hline 1 & 2 & 0 & 8 & 12 & 4.22 \\
\hline 0 & 0 & 4 & 10 & 9 & 4.22 \\
\hline 20 & 2 & 1 & 0 & 0 & 1.17 \\
\hline 0 & 0 & 2 & 3 & 18 & 4.70 \\
\hline 0 & 0 & 0 & 4 & 19 & 4.83 \\
\hline 0 & 0 & 5 & 4 & 14 & 4.39 \\
\hline 11 & 3 & 5 & 0 & 4 & 2.26 \\
\hline
\end{tabular}


Anexo 2. Distribución de las respuestas de priorización del cuestionario tres

Sentencia

1.3 El personal médico no apoya el abordaje de la sexualidad por parte de los profesionales de enfermería por temor a que el usuario abandone la medicación 1.4 Cuando los profesionales de enfermería recibimos alguna demanda relacionada con la sexualidad, tratamos de evitar el tema o derivamos al paciente a otro profesional

$1.5 \mathrm{El}$ abordaje que se realiza en materia de sexualidad es escaso y poco adecuado por falta de formación.

1.6 Los aspectos afectivos y relacionales de la sexualidad se exploran y abordan adecuadamente a diferencia de los aspectos sexuales

2.1 Una de las características que hace de los profesionales de enfermería los adecuados para la exploración de la sexualidad es su accesibilidad y disponibilidad

2.2 Los profesionales de enfermería suelen tener una relación terapéutica directa y continuada con el paciente, lo que favorece la expresión de aspectos íntimos como la sexualidad

2.3 Es habitual que se reaccione a las quejas sexuales de los pacientes de salud mental minimizándolas

2.4 Las habilidades de asertividad, empatía, escucha activa y flexibilidad son fundamentales para la exploración de la sexualidad, siendo el personal de enfermería de salud mental, altamente cualificado en ellas

2.5 Enfermería no tiene competencias para la actuación en este campo, solo debería intervenir si hay una orden médica previa

2.6 Enfermería está presente en todos los dispositivos de salud mental por lo que su cobertura alcanza a la totalidad de los usuarios.

3.1 El abordaje de la sexualidad está muy influido por las creencias y actitudes de los profesionales hacia su propia sexualidad.

3.2 Es importante que exista un vínculo terapéutico sólido entre profesional y paciente para poder realizar el abordaje de aspectos íntimos como la sexualidad

3.3 A día de hoy, existen muchos tabús y prejuicios con respecto al sexo por parte de los profesionales de enfermería.

3.4 Generalmente se minusvalora la importancia de una vida sexual satisfactoria en los pacientes de salud mental.

3.5 Existe tendencia a pensar que, si se pregunta por los problemas de disfunción sexual a los pacientes de salud mental, estos puedan abandonar la medicación.

3.6 Los especialistas de enfermería en salud mental no son los adecuados para abordar la sexualidad porque su formación es escasa.

4.1 La edad es un factor determinante en el abordaje de la sexualidad, reduciéndose significativamente en los extremos.

4.2 Los profesionales nos sentimos más cómodos abordando el patrón sexual con pacientes de nuestro mismo género.

4.3 La edad y el género del paciente no son factores que influyan a la hora de abordar la sexualidad por parte de los profesionales de enfermería.

4.4 Una de las razones por las que no se aborda la sexualidad es porque se tiende a infantilizar a los pacientes de salud mental.

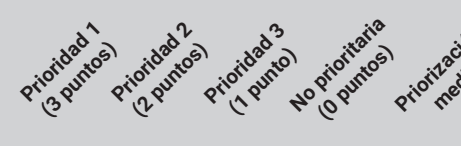

\begin{tabular}{|c|c|c|c|c|}
\hline 0 & 3 & 4 & 12 & 0.52 \\
\hline 1 & 1 & 3 & 14 & 0.42 \\
\hline 7 & 4 & 4 & 5 & 1.74 \\
\hline 2 & 1 & 4 & 12 & 0.63 \\
\hline 2 & 5 & 1 & 11 & 0.89 \\
\hline 8 & 6 & 3 & 2 & 2.05 \\
\hline 4 & 5 & 6 & 4 & 1.47 \\
\hline 5 & 1 & 2 & 11 & 1.00 \\
\hline 0 & 0 & 1 & 18 & 0.05 \\
\hline 0 & 2 & 6 & 11 & 0.53 \\
\hline 2 & 2 & 7 & 8 & 0.89 \\
\hline 5 & 5 & 4 & 5 & 1.53 \\
\hline 3 & 2 & 4 & 10 & 0.89 \\
\hline 9 & 4 & 1 & 5 & 1.89 \\
\hline 0 & 4 & 3 & 12 & 0.58 \\
\hline 0 & 2 & 0 & 17 & 0.21 \\
\hline 10 & 4 & 1 & 4 & 2.05 \\
\hline 5 & 8 & 3 & 3 & 1.79 \\
\hline 0 & 1 & 4 & 14 & 0.32 \\
\hline 0 & 2 & 5 & 12 & 0.47 \\
\hline
\end{tabular}


4.5 Los pacientes con diagnóstico de psicosis son menos preguntados por la sexualidad que pacientes con diagnósticos mentales menos graves. 4.6 Las características del paciente no influyen en el abordaje de la sexualidad. 5.1 No se disponen de herramientas informáticas elaboradas que guíen la exploración de la sexualidad por parte de los profesionales de enfermería. $5.2 \mathrm{La}$ falta de tiempo en la jornada laboral hace que los profesionales de enfermería prioricemos otros aspectos de la enfermedad antes de la sexualidad. 5.3 El tipo de dispositivo en el que desarrollamos nuestro trabajo es el factor contextual que más influye a la hora de abordar la sexualidad.

5.4 Los dispositivos de rehabilitación son los más adecuados para abordar la sexualidad de los pacientes.

5.5 La falta de espacios físicos que garanticen la intimidad y la confidencialidad del paciente hace que no se aborde la sexualidad.

$5.6 \mathrm{El}$ contexto en general no influye en que los profesionales enfermeros aborden o no la sexualidad de los pacientes.

6.1 Los pacientes de salud mental no suelen hablar de sexualidad porque no es un aspecto relevante para ellos.

6.2 Los pacientes de salud mental si demandan atención cuando tienen algún problema de índole sexual.

6.3 La falta de confianza por parte del paciente con su enfermero, hace que no demande atención relacionada con la sexualidad.

6.4 La falta de información de los profesionales a los pacientes sobre sexualidad hace que los pacientes no realicen demandas al respecto.

6.5 Los pacientes de salud mental asumen los problemas con la sexualidad como parte de su enfermedad y no creen que los profesionales enfermeros puedan ayudarlos.

6.6 Una buena relación terapéutica facilita al paciente la expresión de aspectos relacionados con la sexualidad.

7.1 Los pacientes de salud mental establecen relaciones sexuales de riesgo con más frecuencia que la población general.

7.2 Los profesionales de enfermería apenas realizan intervenciones dirigidas a prevenir las conductas sexuales de riesgo en los pacientes de salud mental.

7.3 La educación sexual es la base para evitar conductas sexuales de riesgo en los pacientes de salud mental.

7.4 Es necesario realizar talleres grupales específicos para abordar las conductas sexuales de riesgo.

7.5 El reparto de preservativos en los dispositivos y la información sobre métodos anticonceptivos son medidas eficaces para disminuir las conductas sexuales de riesgo.

7.6 Se podrían reducir los embarazos no deseados en pacientes de salud mental con estrategias de prevención de conductas sexuales de riesgo.

8.1 Para mejorar la atención de los pacientes en materia de sexualidad se debe dar información sobre el tema a todos los pacientes.

8.2 La sexualidad de los pacientes de salud mental no es un aspecto que precise mejoras ya que no es un objetivo en la atención.

8.3 Para mejorar la atención de los pacientes en materia de sexualidad se debe tratar esta como un aspecto más de la valoración integral de los pacientes. 8.4 Se debe dar más formación al personal de enfermería para concienciar de la importancia de la sexualidad en los pacientes.

8.5 Es necesario realizar un trabajo intrapersonal, analizando nuestros propios valores y creencias, para mejorar la atención en sexualidad.

8.6 Hay pocas intervenciones que los profesionales de enfermería podamos desarrollar para mejorar la atención en sexualidad.

\begin{tabular}{|c|c|c|c|c|}
\hline 3 & 4 & 4 & 8 & 1.11 \\
\hline 1 & 0 & 2 & 16 & 0.26 \\
\hline 6 & 4 & 5 & 4 & 1.63 \\
\hline 5 & 5 & 3 & 6 & 1.47 \\
\hline 4 & 3 & 1 & 11 & 1.00 \\
\hline 0 & 1 & 4 & 14 & 0.32 \\
\hline 1 & 6 & 5 & 6 & 1.05 \\
\hline 3 & 0 & 1 & 15 & 0.53 \\
\hline 3 & 1 & 7 & 8 & 0.95 \\
\hline 1 & 0 & 1 & 17 & 0.21 \\
\hline 1 & 3 & 3 & 12 & 0.63 \\
\hline 6 & 6 & 3 & 4 & 1.74 \\
\hline 0 & 4 & 1 & 14 & 0.47 \\
\hline 8 & 5 & 4 & 2 & 2.00 \\
\hline 4 & 5 & 3 & 7 & 1.32 \\
\hline 7 & 4 & 4 & 4 & 1.74 \\
\hline 5 & 6 & 2 & 6 & 1.53 \\
\hline 1 & 2 & 3 & 13 & 0.53 \\
\hline 1 & 1 & 4 & 13 & 0.47 \\
\hline 1 & 1 & 3 & 14 & 0.42 \\
\hline 0 & 4 & 5 & 10 & 0.68 \\
\hline 0 & 2 & 2 & 15 & 0.32 \\
\hline 11 & 4 & 2 & 2 & 2.32 \\
\hline 5 & 8 & 3 & 3 & 1.79 \\
\hline 3 & 1 & 5 & 10 & 0.84 \\
\hline 0 & 0 & 2 & 17 & 0.11 \\
\hline
\end{tabular}

